Draft Version July 10, 2018

Preprint typeset using $\mathrm{IAT}_{\mathrm{E}} \mathrm{X}$ style emulateapj v. 5/2/11

\title{
A CORRELATION BETWEEN Ly $\alpha$ SPECTRAL LINE PROFILE AND REST-FRAME UV MORPHOLOGY
}

\author{
Vivian ${ }^{1,7}$, Shoubaneh Hemmati ${ }^{1}$, Behnam Darvish ${ }^{1}$, Bahram Mobasher ${ }^{1}$, Hooshang Nayyeri ${ }^{1,2}$, Mark \\ Dickinson $^{3}$, Daniel Stern ${ }^{4}$, Hyron Spinrad $^{5}$, Ryan Mallery ${ }^{1,6}$ \\ Draft version July 10, 2018
}

\begin{abstract}
We explore the relationship between the spectral shape of the Ly $\alpha$ emission and the UV morphology of the host galaxy using a sample of $304 \mathrm{Ly} \alpha$-emitting $B V i$-dropouts at $3<z<7$ in the GOODS and COSMOS fields. Using our extensive reservoir of high-quality Keck DEIMOS spectra combined with $H S T$ WFC3 data, we measure the Ly $\alpha$ line asymmetries for individual galaxies and compare them to axial ratios measured from observed $J$ - and $H$-band (restframe UV) images. We find that the Ly $\alpha$ skewness exhibits a large scatter at small elongation $(a / b<2)$, and this scatter decreases as axial ratio increases. Comparison of this trend to radiative transfer models and various results from literature suggests that these high-redshift Ly $\alpha$ emitters are not likely to be intrinsically round and symmetric disks, but they probably host galactic outflows traced by Ly $\alpha$ emitting clouds. The ionizing sources are centrally located, with the optical depth a good indicator of the absorption and scattering events on the escape path of Ly $\alpha$ photons from the source. Our results find no evidence for evolution in Ly $\alpha$ asymmetry or axial ratio with look-back time.
\end{abstract}

Subject headings: galaxies: evolution — galaxies: high-redshift — galaxies: ISM

\section{INTRODUCTION}

Lyman $\alpha$ emission (Ly $\alpha \lambda 1216$ ), due to the electron transition from the second to the ground level of the Hydrogen atom, is often the primary feature available to confirm high-redshift galaxies and to probe their nature. Because of its short wavelength, Ly $\alpha$ is conveniently shifted to optical or near-infrared wavebands at high redshifts and hence, is accessible from ground-based telescopes.

The spectral profile of $\operatorname{Ly} \alpha$ is indicative of the origin and escape path of $\operatorname{Ly} \alpha$ photons, as well as the resonance scattering and extinction by neutral hydrogen and dust in the interstellar medium of galaxies. Specifically, the $\operatorname{Ly} \alpha$ line is often observed to be asymmetric (Stern et al. 1999; Verhamme et al. 2006), double-peaked (Ahn et al. 2001; Tapken et al. 2007; Fosburv et al. 2003; Christensen et al. 2004; Venemans et al. 2005; Kulas et al. 2012; Yamada et al. 2012; Laursen et al. 2011), and/or exhibit a P-Cygni profile (Dickinson 1998; Dey et al. 1998; Bunker et al.|2000; Ellis et al. 2001; Ahn et al. 2003). The different physical origins of Ly $\alpha$ photons from high-redshift star-forming galaxies include hot, ionizing sources (e.g. AGN or massive OB stars in young star clusters or ionized circumstellar regions), cooling from gas heated by shocks from

\footnotetext{
${ }^{1}$ Department of Physics and Astronomy, University of California, Riverside, 900 University Avenue, Riverside, CA 92521, USA; vivianu@ucr.edu

2 Department of Physics and Astronomy, University of California, Irvine, CA 92697, USA

${ }^{3}$ National Optical Astronomy Observatory, Tucson, AZ 85719, USA

${ }^{4}$ Jet Propulsion Laboratory, California Institute of Technology, 4800 Oak Grove Drive, Mail Stop 169-221, Pasadena, CA 91109, USA

${ }^{5}$ Department of Astronomy, University of California, Berkeley, CA 94720, USA

${ }^{6}$ Rebellion Photonics, 7547 South Freeway, Houston, TX 77021 , USA

7 UC Chancellor's Postdoctoral Fellow
}

gravitational collapse, or by shocks due to outflowing gas from starburst regions or AGNs (Yamada et al. 2012). The temperature, density, and motion of the surrounding medium may therefore affect the profile shape of the Ly $\alpha$ emission (Ahn et al. 2001; Tapken et al. 2007). Additionally, processes external to the galaxy, i.e. absorption from the intergalactic medium, may also introduce asymmetry to the line as $\operatorname{Ly} \alpha$ forest opacity increases at higher redshifts (e.g. Rauch et al. 1997). The real situation is likely significantly more complicated, with Ly $\alpha$ emission also depending on the properties of its host galaxy and in particular on its interstellar environment.

To date, several large, dedicated spectroscopic surveys of high-redshift Ly $\alpha$ emitting sources have investigated the profile shape of Ly $\alpha$. Many studies have included quantitative comparison of the Ly $\alpha$ line profile morphologies to predictions from various radiative transfer models (e.g. Verhamme et al.|2006, 2008; Dijkstra et al.|2007; Laursen et al. 2011; Yamada et al. 2012). For instance, Ouchi et al. (2010) studied a sample of $207 z=6.6$ Ly $\alpha$ emitters (LAEs) in the Subaru/XMM-Newton Deep Survey field and found no large evolution of the Ly $\alpha$ line profile from $z=5.7$ to 6.6. A similar finding was confirmed by $\mathrm{Hu}$ et al. (2010) using a sample of $118 z \sim 5.7$ and 6.5 LAEs selected from a narrowband survey of the GOODS-North field. Mallery et al. (2012) investigated the Ly $\alpha$ equivalent widths and escape fractions of 244 sources with Ly $\alpha$ emission at $z \sim 4-6$ and found no redshift dependence; instead, dust extinction is the major factor that inhibits the escape of Ly $\alpha$ photons. These large samples provide a basis for understanding the observed spectroscopic properties of high-redshift LAEs.

In order to further investigate the escape path of Ly $\alpha$ photons from high-redshift LAEs, we explore the link between their spectroscopic and morphological properties, partially motivated by a similar relation studied by Law et al. (2012). Specifically, we examine the relation between Ly $\alpha$ spectral profile shapes and rest-frame 
UV morphologies for a large sample of 304 LAEs (spectroscopically identified by their Ly $\alpha$ line) at $3<z<7$ as well as the evolution of the line profile shapes with redshift. By examining these independent representations of the UV emission, we expect to gain insight into the internal and external processes as well as geometrical and projection effects that may govern the observed shape of Ly $\alpha$ line profiles. We do so by using a spectroscopically selected sample of high-redshift Ly $\alpha$ emitting galaxies in the Great Observatories Origins Deep Survey (GOODS; Giavalisco et al. 2004) and the Cosmic Evolution Survey (COSMOS; Scoville et al. 2007) fields observed with DEIMOS (Faber et al. 2003) on the Keck II Telescope. The available HST ACS and WFC3 images along with multi-band photometric catalogs from COSMOS and the Cosmic Assembly Near-infrared Deep Extragalactic Legacy Survey (CANDELS; Grogin et al. 2011; Koekemoer et al. 2011) provide valuable information regarding the morphology of the rest-frame UV emission.

This paper is structured as follows. The sample selection is described in 92 Section 3 details the Ly $\alpha$ line fitting and morphological analysis of galaxies, while 4 presents the line profile asymmetry and elongation measurements. The implications of our results are given in $\$ 5$ and summarized in 86 , We assume $H_{0}=70 \mathrm{~km} \mathrm{~s}^{-1} \mathrm{Mpc}^{-1}, \Omega_{\mathrm{m}}=0.3$, and $\Omega_{\Lambda}=0.7$ throughout the paper.

\section{SPECTROSCOPIC SAMPLE SELECTION}

The high spectral resolution of the present spectroscopic data set makes it well suited for a study of the Ly $\alpha$ line profile shape. For both GOODS and COSMOS, the high-redshift LAEs have been observed with the optical multi-object spectrograph DEIMOS on the Keck II Telescope. The DEIMOS observations were taken using $1^{\prime \prime}$ slits with either the $600 \mathrm{l} / \mathrm{mm}$ or the $830 \mathrm{l} / \mathrm{mm}$ gratings (full width half maximum resolution of $3.5 \AA$ and $2.5 \AA$, respectively) spanning a wavelength range $\sim 5,000-10,000 \AA$, designed to detect Ly $\alpha$ lines at high redshifts. Given the redshift range of our sample $(3.22<z<7.21)$, the spectral resolution at $\operatorname{Ly} \alpha$ is $R=1470-4000$, corresponding to velocity resolution $\Delta V=75-200 \mathrm{~km} \mathrm{~s}^{-1}$. Integration times per mask varied, but was typically on the order of two hours per mask.

\subsection{The GOODS Sample}

The Keck spectroscopic follow-up campaign of highredshift galaxies (largely selected as Lyman break galaxies, or LBGs, as well as from photometric redshifts and narrowband surveys) in the GOODS+CANDELS fields has been an on-going project since 2005. We used the DEEP2 pipeline to process the DEIMOS data. The observed 2D spectra were sky line subtracted and flat fielded. The observed standard lamps were used to construct a wavelength solution and to wavelength calibrate the spectra. The individual $1 \mathrm{D}$ spectra were extracted from the reduced $2 \mathrm{D}$ using nominal positions from the mask design files using boxcar and optimal extractions. For the $830 \mathrm{l} / \mathrm{mm}$ grating, observations were dithered to correct for the ghost effect and for better skyline subtraction. In this case, a modified version of the pipeline was used where the data were processed using the dithering pattern recovered by the pipeline from observations of the bright stars on the mask. The final output are 1D and 2D spectra for individual sources, wavelength calibrated and skyline subtracted.

All the reduced spectra in the GOODS sample have been visually inspected by at least two experienced team members for redshift identification. From the pool of sources at the appropriate redshift range where Ly $\alpha$ is observed, we applied a detection limit of $3 \sigma$ in Ly $\alpha$ line flux which results in the selection of 130 LAEs with secure (quality code $Q=B$; well-identified Ly $\alpha$ line) and very secure $(Q=A$; well-identified $\operatorname{Ly} \alpha$ and additional emission or absorption lines) redshifts. Ninety-one of the LAEs are in the GOODS-North field (with only five sources matched to AGN identified in a 2-Megasecond X-ray catalog; private communication with D. Kocevski), while 39 are in the GOODS-South field. These sources were matched to the CANDELS photometric catalog and encompass the sample of LAEs from GOODS considered in this study.

The LAEs were further categorized by their Lyman break properties. Previous photometrically selected samples have allowed us to probe the UV luminosity function at $4<z<8$ (e.g. Bouwens et al. 2010) and raise questions about the reionization state of the intergalactic medium (IGM). Since the Ly $\alpha$ emission fraction in LBGs depends on the IGM neutral fraction (Stark et al. 2011), it is crucial to clarify the spectral properties of Ly $\alpha$ in LBGs as identified by dropout techniques. Dropout techniques utilize images taken in multiple photometric wavebands to detect the Lyman break in high-redshift sources (Steidel et al. 1996; Dickinson 1998). For instance, the following colorcolor selection as adopted from, e.g. Beckwith et al. (2006) and Stark et al. (2009), are ideal for identifying candidate star-forming galaxies at $z>3$ :

$B$-dropouts:

$$
\begin{aligned}
B_{435}-V_{606}>1.1+ & V_{606}-z_{850}, \\
& B_{435}-V_{606}>1.1, \\
V_{606}-z_{850} & <1.6, \\
S / N\left(V_{606}\right) & >5, \\
& \text { and } S / N\left(i_{775}\right)>3 .
\end{aligned}
$$

$V$-dropouts:

$$
\begin{gathered}
V_{606}-i_{775}>1.47+0.89\left(i_{775}-z_{850}\right) \quad \text { or } 2, \\
V_{606}-i_{775}>1.2, \\
i_{775}-z_{850}<1.3, \\
S / N\left(z_{850}\right)>5,
\end{gathered}
$$

and $\quad S / N\left(B_{435}\right)<3, \quad$ or $\quad B_{435}-i_{775}>V_{606}-i_{775}+1$.

$i$-dropouts:

$$
\begin{aligned}
& i_{775}-z_{850}>1.3, \\
& \qquad / N\left(z_{850}\right)>5, \\
& \text { and } S / N\left(V_{606}\right)<2, \text { or } V_{606}-z_{850}>2.8 \text {. } \\
& \text { Using the available photometry catalogs for }
\end{aligned}
$$


GOODS (Guo et al. 2013, Barro et al. in prep.), we categorize the LAEs by their Lyman break properties. The LBG color selection thus results in $57 B$-dropouts, $20 \mathrm{~V}$-dropouts (one of which is also a $B$-dropout), and $6 i$-dropouts. The remaining 47 sources fail to qualify as a dropout due to one of two reasons: either photometric errors in their broad-band photometry scatter them in color-color space, or that the $\operatorname{Ly} \alpha$ equivalent width is so large it affects their broad-band colors used to determine their LBG status. The LAEs are shown on the color-color diagrams in Fig. 1.

\subsection{The COSMOS Sample}

The COSMOS sample of LAEs were initially presented in Mallery et al. (2012), with similar spectroscopic observational setups and reduction procedures as the GOODS sample. These sources were selected to have photometric redshifts $z>3.8$ and were complete to $z$ magnitude $z^{+}<25$ and mass $>10^{10.5} M_{\odot}$ (Mallery et al., in prep; Capak et al., in prep). Narrowband selections were also applied to identify LAEs (Scarlata et al., in prep; Shiova et al. 2009; Muravama et al. 2007). Of the 244 objects from Mallery et al. (2012), 210 were matched to the available COSMOS morphology and photometry catalogs (Leauthaud et al. 2007; Capak et al. 2007). Recent DEIMOS observations added 18 new LAEs, and altogether there were 174 sources that met the 3- $\sigma$ Ly $\alpha$ detection criteria. Adopting the LBG selection in $\S 2.2$ of Mallery et al. (2012), 9 were $B$-dropouts, 23 were $V$ dropouts, and one was an $i$-dropout.

\subsection{Properties of the Combined Sample}

Given the consistency between the observations and analyses of the GOODS and COSMOS LAEs, we combine the two populations for a final sample of 304 LAEs (including $66 \mathrm{~B}$-dropouts, $43 \mathrm{~V}$-dropouts - one of which is also a $B$-dropout, and $7 i$-dropouts). The redshift distribution is shown in Fig. 2, spanning the range $3.22<$ $z<7.21$ with a median $\langle z\rangle=4.55$. Properties of the entire sample of LAEs can be found in Table 1, while Table 2 gives a summary of the dropouts. We note that while the observational set up and analyses of the GOODS and COSMOS sources are consistent, the selections of the Lyman-break dropouts likely differ slightly for the two fields. The selection of LBGs in the GOODS fields was based primarily on existing multi-waveband images taken in the HST ACS bands (e.g. Vanzella et al. 2009), whereas the selection in COSMOS relied on a combination of photometric filters used in the Subaru Deep Survey (e.g. Ouchi et al. 2004) and narrowband filters (see Mallery et al. 2012, for details). The depths in the equivalent bands between the two fields might also be different. In this paper we do not directly compare the different selection criteria used for categorizing LBGs. We merely present their properties for the aggregate sample, and encourage curious readers to extract and consider the properties of those dropouts from their survey of choice.

We also point out that there are 190 sources classified as 'Other' in our sample: these are Ly $\alpha$-emitting sources not classified as a dropout. The non-dropouts in GOODS are likely too faint for color selection, whereas those in COSMOS were primarily selected in narrowband surveys. Thirty-nine of these 190 sources are not detected in $z^{\prime}$-band, whereas all dropouts are detected. At $B$-band, the non-detection percentage among the nondropouts reaches $35 \%(106 / 304)$, whereas that for the dropouts is only $18 \%(20 / 114)$. A detailed comparison between these galaxies and the LBGs will be presented in a forthcoming paper; we explore their similarities and differences briefly here.

\section{ANALYSIS}

\subsection{Emission Line Fitting}

All DEIMOS spectra were individually inspected following a detailed procedure outlined in Mallery et al. (2012). As this is a spectroscopically selected sample, Ly $\alpha$ emission is seen in all the sources and has been used for redshift determination. We fitted the line profile of Ly $\alpha$ with a skewed Gaussian, and kept sources with at least a 3- $\sigma$ detection in Ly $\alpha$ flux over the noise in the continuum. The functional form of the skewed Gaussian was adopted from Mallery et al. (2012) and reproduced here:

flux $=A \times e^{-0.5 \times((\lambda-x) / \omega)^{2}}\left(\int_{-\infty}^{s(\lambda-x) / \omega} \exp \left(-t^{2} / 2\right) d t\right)+c$.

The fit returns values for the flux normalization $(A)$, the first moment of a standard Gaussian $\left(\lambda_{0}=x+\right.$ $\omega \delta \sqrt{2 / \pi}$ ), the second moment of a standard Gaussian $\left(\sigma=\omega \sqrt{1-2 \delta^{2} / \pi}\right)$, the value of the skew $(s)$, and the value of the continuum $(c)$, where $\delta=s / \sqrt{1+s^{2}}$. For a symmetric line, skew $s$ would be 0 . The sign of skewness demonstrates the directionality of the asymmetry, e.g. a large skew value indicates an asymmetric Ly $\alpha$ line with a large red wing, and a negative skewness denotes a blue wing. Fig. [3illustrates some examples of the skewed and non-skewed Ly $\alpha$ lines at the lower signal-to-noise threshold (SNR $\lesssim 5$ ) along with their corresponding fits. An in-depth analysis to test the robustness of our measurements from line-fitting is presented in the Appendix. We report here the skewness and its associated uncertainty from the fit, as this quantity best describes the asymmetry of the Ly $\alpha$ line profile and could be used to trace the escape path of Ly $\alpha$ photons (Yamada et al. 2012).

\subsection{Morphological Analysis}

To investigate the morphology of the galaxies from which Ly $\alpha$ photons escape, we examine the rest-frame UV emission using the HST WFC3 F125W and F160W ( $J$ and $H$ bands, respectively, hereafter) images from CANDELS. Given the redshift range of our sample (3.22 $<z<7.21)$, we divided it into two bins $(z<5$ and $z>5)$ such that the observed $J$ - and $H$-band images correspond to the same rest-frame $\sim 2300 \AA$ emission in the low and high redshift bins, respectively. This helps to minimize any effect in the geometry of the galaxy that might be due to the change in bandpass. For galaxies above redshifts $z \gtrsim 5.5$, the choice of WFC3 filters over ACS ones is necessary to avoid having Ly $\alpha$ in the waveband, despite the better angular resolution due to smaller point spread function (PSF) full width half maximum and less undersampling afforded by ACS. In addition, the $J$ - and $H$-bands should not contain any 

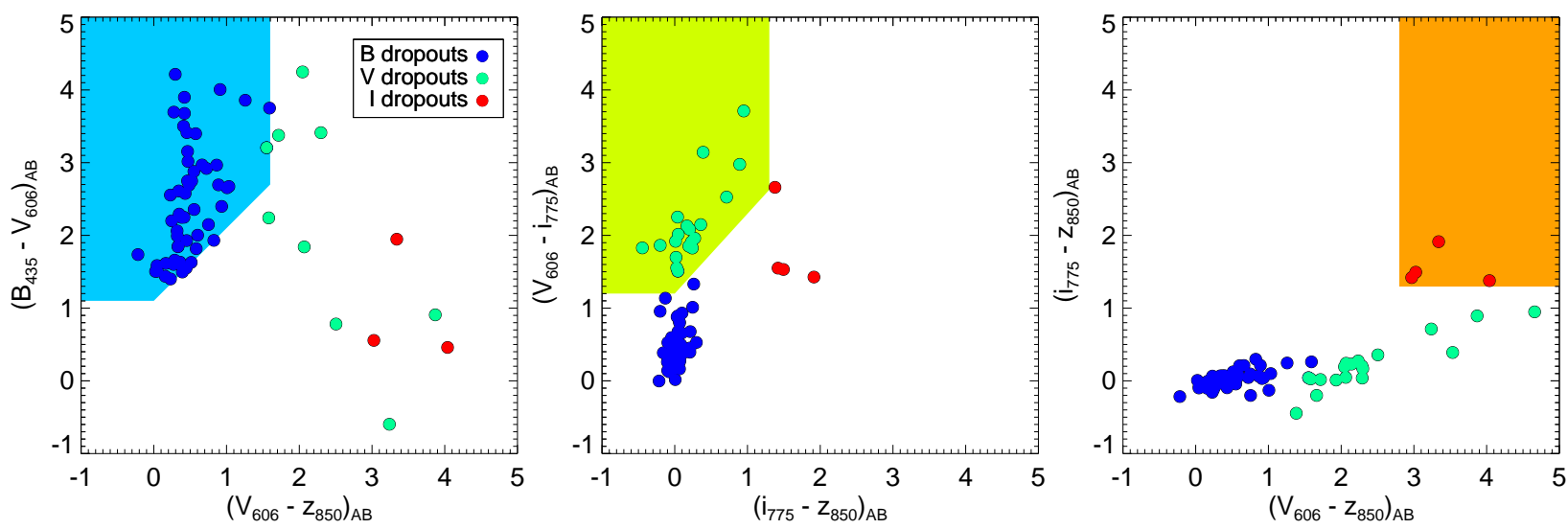

Figure 1. Color-color diagrams for the LAE sample in the GOODS fields, showing LBG color selection of $B V i$ dropouts as indicated by the shaded areas in each panel. Of the 130 sources identified with the Ly $\alpha$ line (black circles) in GOODS, 61 are categorized as $B$-dropouts (blue circles), 21 as $V$-dropouts (green circles; one of which is also a $B$-dropout), and eight as $i$-dropouts (red circles).

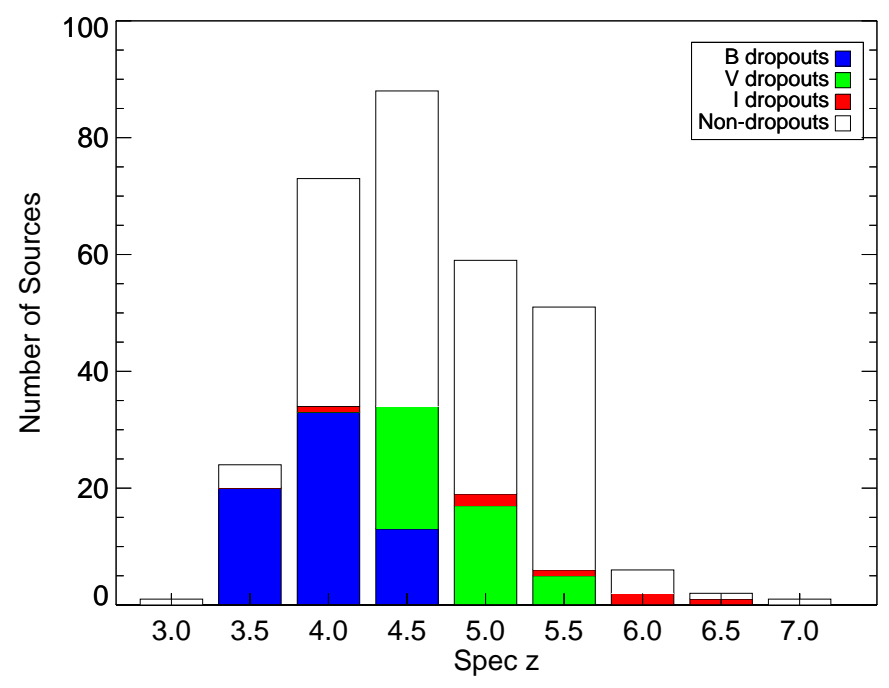

Figure 2. The stacked redshift distributions of the $B$-dropouts (blue), $V$-dropouts (green), $i$-dropouts (red), and non-dropouts (white) with bin sizes of 0.5 . Combined, the final sample spans the redshift range of $3.22<z<7.21$ with median $\langle z\rangle=4.55$.

significant line emission, and so they allow for pure measurements of the stellar continuum morphology. In contrast, the [OIII] line at $5007 \AA$ has been shown to be a common source of contamination in high-redshift LBGs and LAEs (Stark et al. 2013; Schenker et al. 2013; Pénin et al. 2015), particularly for those sources with inferior quality flags. Therefore, we settled on using WFC3 images for our morphological analysis for consistency and reliability (e.g. Mosleh et al. 2012). We focus, in particular, on the axial ratio, or elongation, as a proxy for the morphology of the rest-frame UV emission. The shape of the galaxy, as described by the axial ratio, has implications for the viewing angle and, consequently, the amount of obscuration escaping Ly $\alpha$ photons encounter along our line of sight.

Using the WFC3 images from CANDELS, we ran Source Extractor over the near-infrared images to extract the axial ratios of the galaxies. Unfortunately, equivalent
WFC3 images are not available for the COSMOS field outside of the CANDELS area where much of the current LAE sample reside. For the COSMOS fields, we have available HST ACS F814W ( $I$ band hereafter) images for all LAE sources, and extracted the relevant mrophology from Leauthaud et al. (2007). Thus, we calibrate $I$-band Source Extractor (Bertin \& Arnouts 1996) measurements by comparing the same $I$-band measurements to their $J$ - and $H$-band counterparts in GOODS-North.

Results in Fig. 4 4 show that the offsets in $I$ - vs. $J$-band and $I$ - vs. $H$-band increase for larger elongations, and are almost negligible for smaller elongations. The mean scatter is smaller for our LAE sample $\left(\overline{\delta_{i j}}=0.127\right.$ and $\left.\overline{\delta_{i h}}=0.134\right)$ than for the rest of the detected sources in GOODS-N $\left(\left\langle\delta_{i j}\right\rangle=0.133\right.$ and $\left.\left\langle\delta_{i h}\right\rangle=0.142\right)$. We note that the linear regression line for the detected sources in GOODS-N has a shallow slope $(<1)$, which is expected due to the PSF difference between WFC3 and ACS. The effect is pronounced at high axial ratios, where galaxies that are more elongated would look "rounder" in the WFC3 data due to the larger PSF. The underlying population also includes galaxies at lower redshifts (e.g. $z<2$ ) where one might expect rounder shapes if WFC3 samples the optical rest-frame compared to UV-rest frame for ACS, since rounder bulges become more prominent at redder wavelengths. This effect does not apply to the high-redshift LAEs in our sample (where only the aforementioned PSF effect would dominate), and thus the fit to our LAEs have a steeper slope compared to that for the underlying GOODS-N population, but still a shallow slope $(<1)$. Thus, the relations allow us to calibrate the $I$-band axial ratios $(a / b)$ to that in $J$ - and $H$-bands for the COSMOS sources as follows:

$$
\begin{aligned}
& a / b_{F 125 W}=0.24+a / b_{F 814 W} \times 0.79 \\
& a / b_{F 160 W}=0.29+a / b_{F 814 W} \times 0.75
\end{aligned}
$$

\section{PROPERTIES OF HIGH-REDSHIFT Ly $\alpha$ EMITTERS} 4.1. Lya Line Profile and Galaxy Morphology 

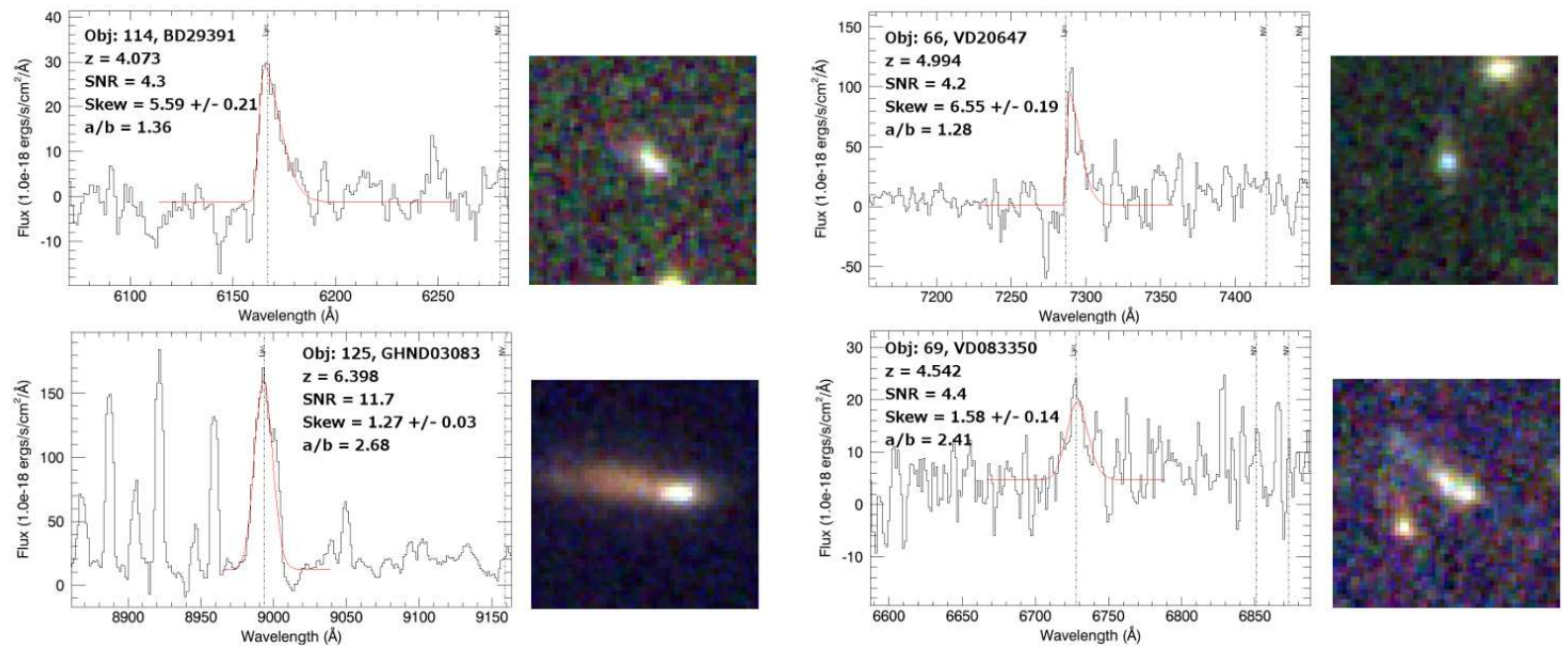

Figure 3. Examples of fitted Ly $\alpha$ lines along with their $z J H$-image in pseudo-RGB colors. The objects are selected to illustrate the span of skewness and axial ratio.
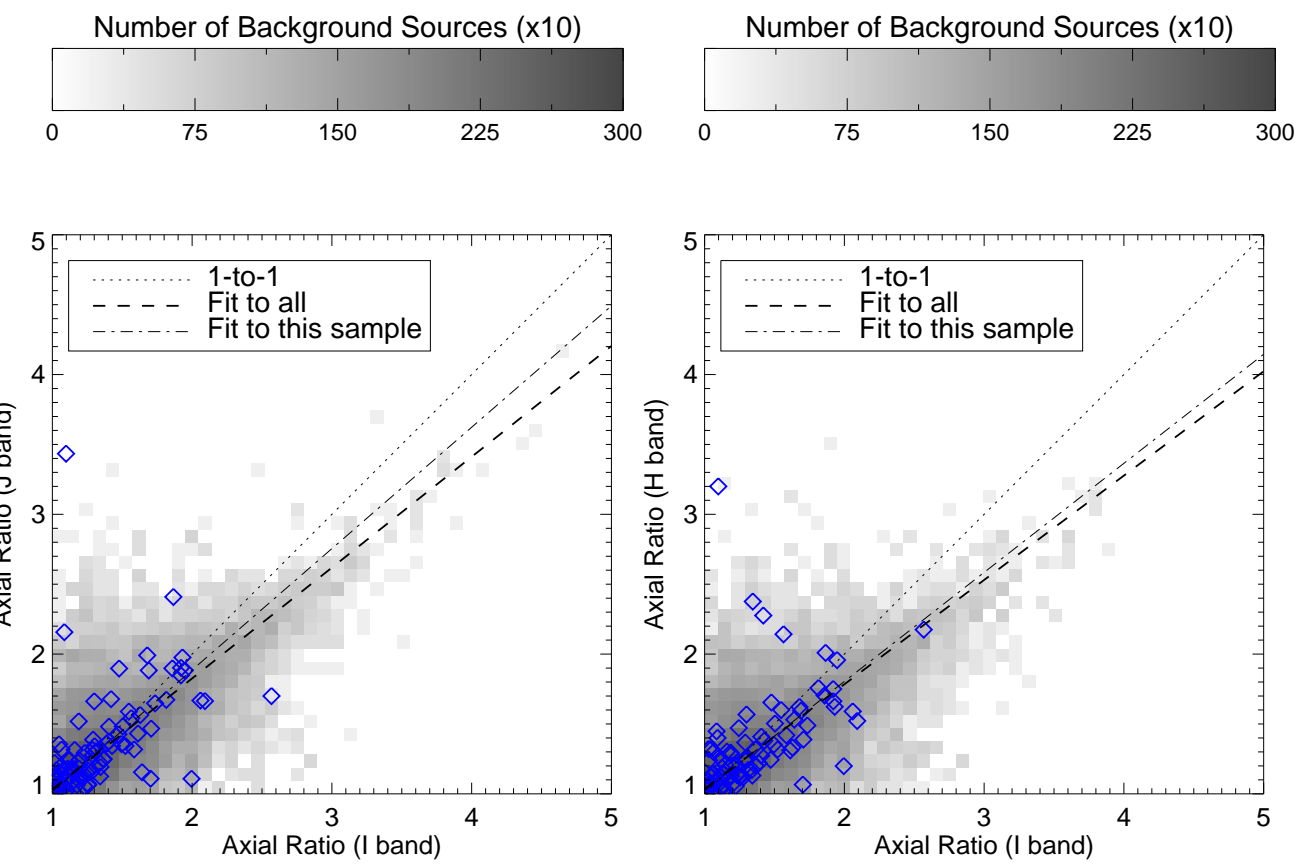

Figure 4. Comparison of the axial ratio measured in the $I$-band versus the $J$-band (left) and $H$-band (right) for all detected sources in the GOODS-N field. The blue open diamonds represent the LAEs in this sample. The correlations are good between the bands, with negligible offset at the small end gradually increasing with elongation. Our sample (blue diamonds) exhibits smaller scatter $\left(\overline{\delta_{i j}}=0.127\right.$ and $\left.\overline{\delta_{i h}}=0.134\right)$ compared to the rest of the population $\left(\overline{\delta_{i j}}=0.133\right.$ and $\left.\overline{\delta_{i h}}=0.142\right)$.

We examine the relation between the Ly $\alpha$ spectral profile shape and the morphology of the rest-frame UV emission (as measured from the axial ratios) of the LAEs in Fig. 5. For this analysis, we plotted all 304 LAEs in our sample, LBG or not, as black circles, and sub-populations of $B$-dropouts, $V$-dropouts, and $i$-dropouts are colored in blue, green, and red, respectively. For low axial ratio values $(a / b<\langle a / b\rangle=1.26)$, a large dispersion in skewness $(\sigma=1.44)$ is found. As the elongation of the galaxies increases, this dispersion decreases $(\sigma=1.27$ for $a / b>1.26)$ and the skewness of $\operatorname{Ly} \alpha$ converges to
1.53. More importantly than the measured dispersions, a large tail of galaxies with high skewness is observed. No sources with large axial ratio exhibits large skewness in their Ly $\alpha$ spectral profile shape. The physical interpretation of this result is discussed in \$5.

The skewness distribution of LAEs is shown on the right panel of Fig. 5. The median skewness of the dropouts is $0.03-0.26$ elevated compared to the entire sample, but otherwise the distributions are similar (see Table 2). We employ the Kolmogorov-Smirnov (KS) test to determine the probability that the dropout and 
non-dropout populations are drawn from a similar distribution. Results from the KS tests among the various dropout groups return $p$-values of $0.15-0.97$, so any difference between these distributions is far from significant.

We consider how our sample's skewness distribution compares with that from Yamada et al. (2012), who presented the Ly $\alpha$ line profile shapes of 91 emitters at $z=3.1$. While we are unable to make a direct quantitative comparison between our asymmetry measurement with their symmetry index, Yamada et al. (2012) found that 27 of their objects are asymmetric ("strong red and weak blue"), though at least half are only moderately so. Only three of their objects have blue wings ("strong blue and weak red"), whereas the rest of their sample are slightly to moderately asymmetric. These results are qualitatively consistent with our observations that the majority of our sample are slightly asymmetric $(0<s<2)$ with a tail trailing off to high skewness.

The bottom panel in Fig. 5 illustrates the distribution in axial ratio for the LAEs. Again, the distributions appear similar across the subgroups. The median axial ratio decreases slightly from $B$-dropout $(\langle a / b\rangle=1.22)$ to $V$-dropout $(\langle a / b\rangle=1.21)$ and $i$-dropout $(\langle a / b\rangle=1.17)$, but this may well be due to small number statistics since only $9 i$-dropouts were identified in the sample. Indeed, the KS tests for the axial ratios measured for the different sub-groups have $p$-values of $0.60-0.83$, suggesting that within the limits of our tests, the distributions are not significantly different.

We further consider the observed axial ratio distributions of LBGs at these redshifts. Ferguson et al. (2004) reported that the ellipticity distribution for a sample of $z \approx 4 B$-dropouts resembled that of flattened disks, reasoning that many of the LBGs have several concentrations of light spread across the galaxy instead of a nucleated concentration. The mean ratio they derived is $a / b=1.54$, higher than that for our $B$-dropouts. The discrepancy between our measurements is likely due to the difference in sample selection. The Ferguson et al. (2004) sample of LBGs include LAEs as well as nonemitters, and the latter likely suffer from severe optical depth, absorption, or radiative transfer effects. Therefore, it is sensible that our LAEs would exhibit a lower typical axial ratio and appear more spheroid-like than an LBG sample that incorporates emitters and non-emitters alike. How this optical depth effect may play into the relation between axial ratio and morphology will be revisited in $\$ 5.2$

\subsection{Evolution with Redshift}

2 illustrates some differences in skewness and axial ratio among the $B V i$-dropouts, and this distinction raises the question of whether or not redshift-dependent factors such as IGM absorption and diminishing sensitivity have an effect on the Ly $\alpha$ profile shape and morphology. In order to investigate if the trend of decreasing dispersion in skewness as a function of axial ratio is due more to intrinsic properties than redshift, we plot each of these properties as a function of redshift in Fig. 6. The full sample as well as median values of axial ratio and skewness within each redshift bin are plotted. We perform a Spearman rank correlation test as a nonparametric measure of potential statistical dependence between each of these properties with redshift, and determined $\rho_{a / b}=0.05(p$-value $=0.41)$ and $\rho_{\text {Skew }}=-0.004(p$ value $=0.94)$. As such, we find no evolutionary trend with either property. Additional discussion on how IGM may affect the Ly $\alpha$ line profile can be found in $\$ 5.3$.

Another check for the validity of the trends in axial ratio and skewness regards their correlation with the signalto-noise ratio (SNR) cut applied to the initial selection of the LAE sample. We focus on a subset of our LAEs in the COSMOS field (Mallery et al. 2012) with proper flux calibrations for this test. We observe a relatively flat distribution of source radii as a function of Ly $\alpha$ line SNR in Fig. 7] with Spearman rank coefficient $\rho_{\text {radius }}=-0.01$ and $p$-value $=0.89$. We note an increase in Ly $\alpha$ line flux correlated with the sources with $\mathrm{SNR} \gtrsim 6\left(\rho_{\text {flux }}=0.34\right.$ with $p$-value of $\left.4.04 \times 10^{-6}\right)$, but no strong trends can be seen in skewness $\left(\rho_{\text {Skew }}=0.13\right.$ with $p$-value of 0.08$)$ or axial ratio $\left(\rho_{a / b}=-0.01\right.$ with $p$-value of 0.92$)$ with Ly $\alpha$ line SNR.

\section{DISCUSSION}

In this section we draw on the observed trends in skewness and axial ratio to infer the physical mechanism and geometric or projection effect for the propagation of Ly $\alpha$ photons. We start with the simplifying assumption that these star-forming galaxies are intrinsically round, symmetric disks. We note that while we treat the axial ratio as a proxy for the viewing angle of the galaxy, it would not be a perfect correlation without kinematics data to help disentangle the intrinsic inclination angle of the galactic disks. Another caveat is that although many of the sources with low axial ratio may simply be compact, the fraction of sources with low axial ratios (e.g. $a / b<1.5$ ) but which have half-light radii $r>0$.' 15 is nontrivial, $\sim 24 \%$. Thus, we do consider the case where the axial ratio may reflect the viewing angle of the galaxy. In the following sections, we discuss two plausible scenarios for the origin of the Ly $\alpha$ photons, and explore how the line profile shape would vary in each case in the context of our observations. The overall impact from the IGM is also examined.

\subsection{Outflows in High-Redshift LAEs}

Outflows are known to be commonplace in highredshift star-forming systems (Pettini et al. 2001; Frve et al. 2002; Vanzella et al. 2009). In particular, many studies have presented observational spectroscopic signature consistent with outflows and expanding shells in $z \sim 2-3$ LBG samples (e.g. Shapley et al. 2003; Steidel et al. 2010; Kulas et al. 2012). It has been shown that the Ly $\alpha$ profile shape depends on the expanding velocity of the shell (Verhamme et al. 2006), though this prediction is not currently tested for our sample until the systemic velocity is measured independently from the Ly $\alpha$ line. Here we consider the effect of interstellar and circumgalactic gas kinematics on the Ly $\alpha$ photons and galaxy viewing angle.

If the Ly $\alpha$ emitting hydrogen clouds were behind outflowing gases perpendicular to the galactic disk, the broadening of the Ly $\alpha$ line together with intergalactic absorption could cause the line to appear asymmetric. Models from Verhamme et al. (2006) have shown that in the case of an expanding shell with a central monochromatic source, the more backscatterings the Ly $\alpha$ photons undergo on their escape path, the more the red 


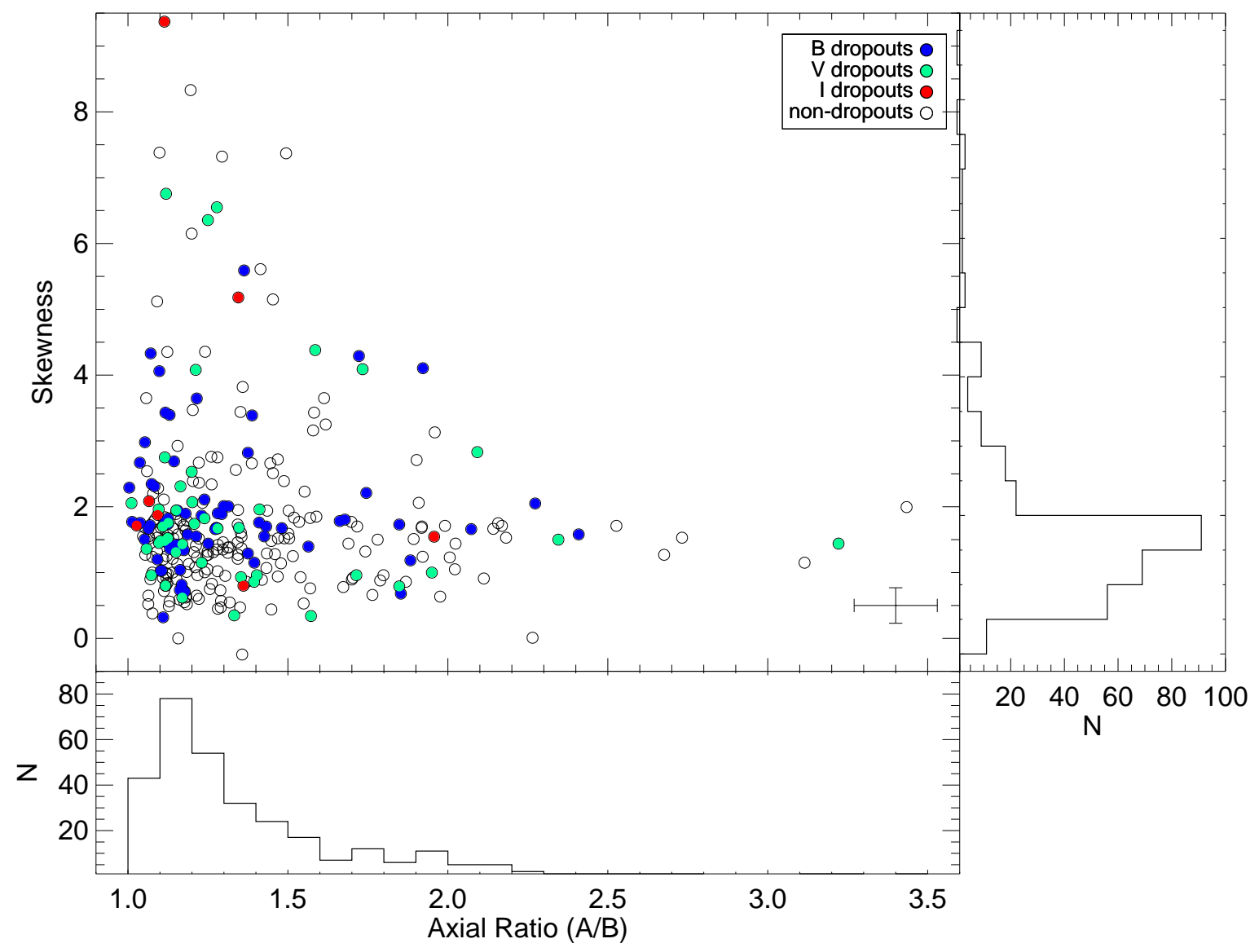

Figure 5. Skewness as a measure of the asymmetry of the Ly $\alpha$ emission line is plotted against axial ratio for all the LAEs (black). Subpopulations are color-coded by the dropout colors: $B$-dropouts (blue), $V$-dropouts (green), and $i$-dropouts (red). Typical error bars are shown in the lower right. The dispersion in skewness decreases from $\sigma=1.44$ for $a / b<1.25$ to $\sigma=1.27$ for $a / b>1.26$ as elongation increases for the population. The distribution in skewness and axial ratio are illustrated on the right and bottom, respectively.

wing will be broadened in the line profile. This effect is most pronounced in the face-on cases where we observe the outflowing gas along our line of sight, and is negligible if the outflow velocity vector is perpendicular to our line of sight. This is consistent with the trend seen in Fig. 5, suggesting that the physical mechanism motivating the observed Ly $\alpha$ line profile in the context of the galaxy elongation may be outflows. At low redshifts, Kornei et al. (2012) found a correlation between inclination and outflow velocity for a sample of $z \sim 1$ star-forming galaxies, while Law et al. (2012) found none for their $z \sim 2$ sample. An interesting follow-up question for the subset of high $\mathrm{S} / \mathrm{N}$ sources in our sample is how the outflow velocities relate to Ly $\alpha$ profile and UV morphology.

\subsection{Rotation, Viewing Angle, and Optical Depth}

We next consider the case where the Ly $\alpha$ emission emerges from the galactic disk, in the absence of outflows. In a recent paper, Garavito-Camargo et al. (2014) presented the results of radiative transfer calculations measuring the impact of gas bulk rotation on the line profile shape. The fraction of Ly $\alpha$ photons that escape at various velocities (as a proxy for the Ly $\alpha$ line profile shape) is shown for different rotations and viewing angles. A double-peaked emission line may become single- peaked when the photon-emitting source has large rotation velocity $\left(V=200-300 \mathrm{~km} \mathrm{~s}^{-1}\right)$. This effect is most pronounced for the edge-on view as well as low neutral hydrogen optical depth. An additional effect that may complicate the line morphology is the absorption blueward of the Ly $\alpha$ peak by intergalactic gas clouds. (A more detailed discussion of IGM effects is in \$5.3.) Thus, a Ly $\alpha$ line that should otherwise have been doublepeaked would now appear asymmetric, e.g. in the case of fast rotation $\left(V>200 \mathrm{~km} \mathrm{~s}^{-1}\right)$ at low viewing angle. It becomes single-peaked in the case of minimal rotational velocity, regardless of the viewing angle. This can be seen in both Figures 4 and 5 of Garavito-Camargo et al. (2014).

While these models may explain the relatively symmetric single-peaked lines for sources with high viewing angle and minimal rotation, they underpredict the asymmetric lines observed at low axial ratios, nor do they sufficiently explain the lack of asymmetric profile shapes at high axial ratios. To address these issues, we further consider the optical depths probed in these galaxies. The sources with low axial ratios are likely to exhibit lower optical depths as viewed face-on, contrary to those that appear edgeon. Looking at the models from Garavito-Camargo et al. (2014), if the viewing angle is held constant, say, in the 


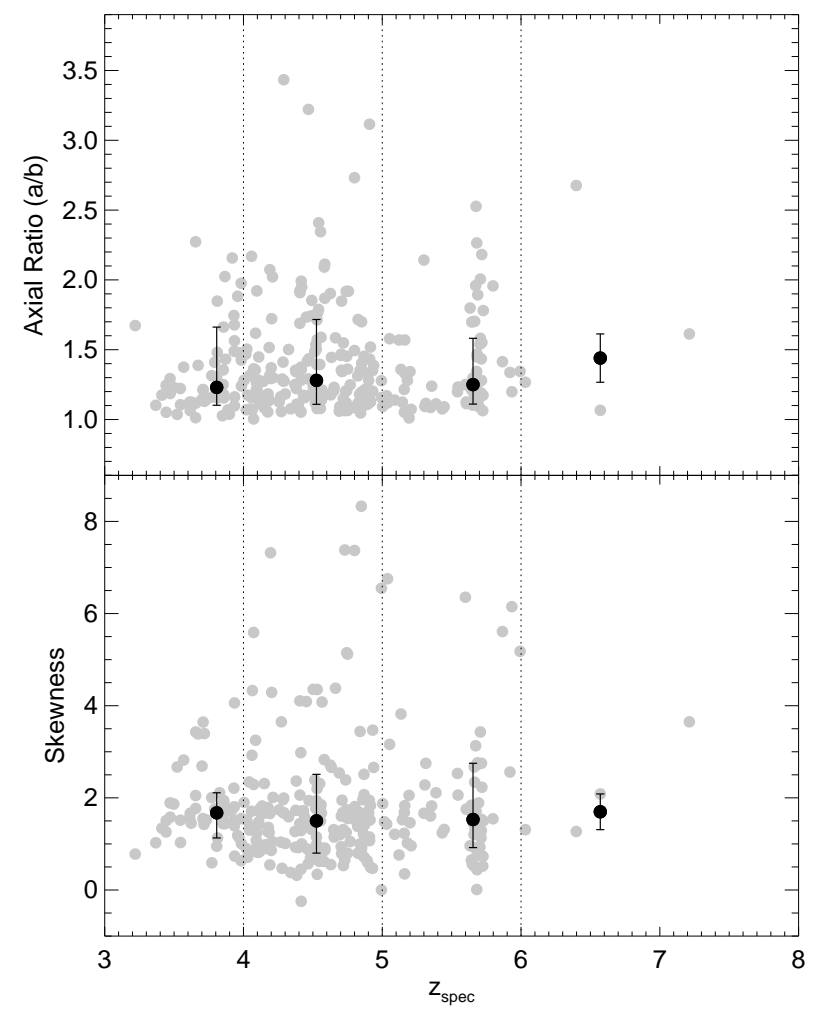

Figure 6. Axial ratio (top) and skewness (bottom) of the LAEs plotted as a function of redshift in different redshift bins (as segregated by the dotted lines). The grey points are from the full sample, overlaid by black filled circles that denote the median values of the axial ratio and skewness within each redshift bin. Vertical error bars illustrate the ranges where $68 \%$ of the sources reside. No evolutionary trend is observed with either property, with Spearman rank coefficients $\rho_{a / b}=0.05(p$-value $=0.41)$ and $\rho_{\text {Skew }}=-0.004$ $(p$-value $=0.94)$.

face-on case (e.g. $|\cos (\theta)|=1$ in their Fig. 2), the red part of the line would become more and more symmetric as the optical depth increases from $10^{5}$ to $10^{7}$. This may be due to the fact that, in the optically thick case, the Ly $\alpha$ photons are more likely to scatter into the wing of the line and appear double-peaked. In this light, the red part of the Ly $\alpha$ line would appear more symmetric at high $a / b$ values, leading to the relative lack of sources at the high axial ratio - high skewness parameter space.

\subsection{Absorption by the Intergalactic Medium}

The effect of IGM absorption on the line profile of Ly $\alpha$ has been examined and modeled at length by previous studies. For instance, Laursen et al. (2011) computed the line profile shape as well as the escape fractions of Ly $\alpha$ photons for a suite of simulated galaxies with different circular velocities. While the Ly $\alpha$ line asymmetries was not explicitly quantified, the study investigated the relative strength of the two peaks in Ly $\alpha$ as viewed through different IGM conditions at various redshifts. The result that the blue peak is often weak, if not completely absorbed, at $z>3.5$. This is consistent with our sample having very few double-peaked sources and mostly strong-red-peak sources. The Laursen et al. (2011) models show that some absorption on the blue side of the red peak may be due to the IGM, which could

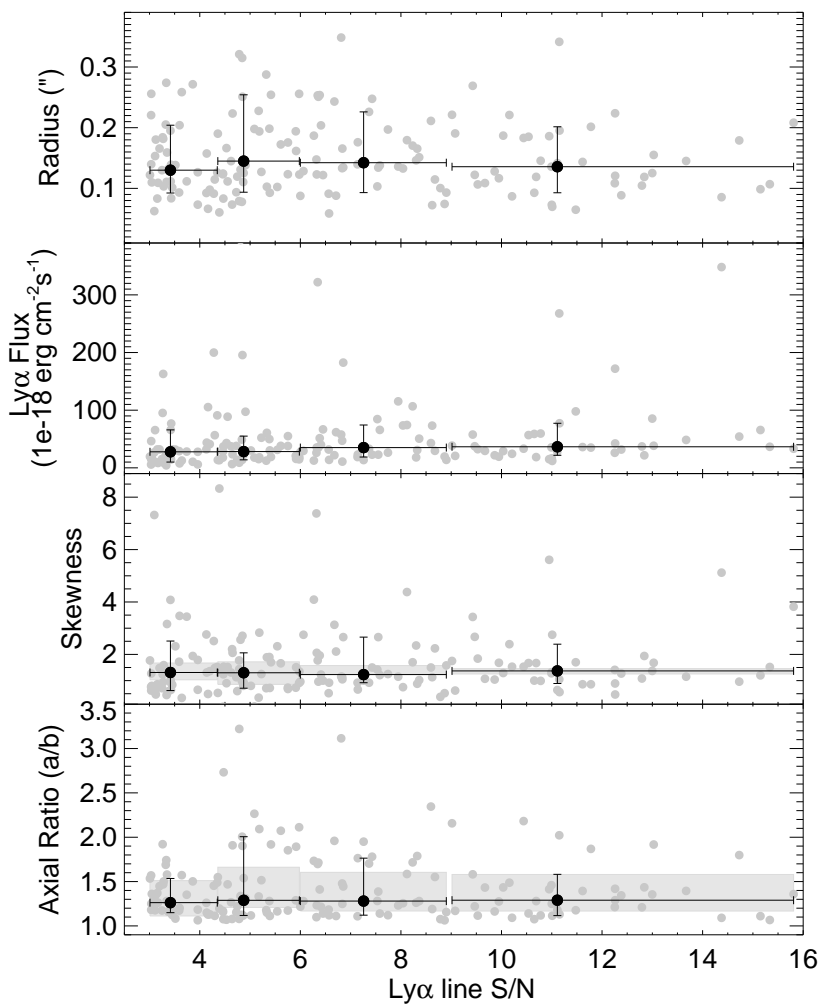

Figure 7. (Top to bottom) Source radius, Ly $\alpha$ flux, skewness, and axial ratio plotted as a function of $\mathrm{S} / \mathrm{N}$ in Ly $\alpha$ line flux for the subsample of COSMOS LAEs with flux calibrations. The bins in SNR are equal in numbers, with their ranges represented by the horizontal error bars. Median values along with vertical error bars highlight the ranges where $68 \%$ of the sources reside. In the bottom two panels, the grey rectangles represent the typical $\pm 1-\sigma$ uncertainties in skewness and axial ratio, respectively, on individual data points centered on the mean value of each bin. The error on axial ratios were not directly output from Source Extractor, but rather represent the propagated results on typical errors from Source Extractor measurements (Ravindranath et al. 2006).

be responsible for the skewness we observed. However, many other factors (e.g. outflows) may be in play such that the profile shape, and hence Ly $\alpha$ asymmetry, would not necessarily increase with redshift, which is consistent with what we observed in Fig. 6.

Dijkstra et al. (2007) also studied the impact of IGM on the profile shapes of high-redshift Ly $\alpha$. Using a skewness parameter originally defined by Kashikawa et al. (2006), Dijkstra et al. (2007) find that the observed shape of $\operatorname{Ly} \alpha$ may vary widely depending on the star formation rate, ionizing background, and the intrinsic width and systemic velocity of the line. In particular, they find that galaxies with large star formation rates or those embedded in a strong ionizing background tend to display symmetry in the Ly $\alpha$ line, at least near the low end of our redshift range. As our sample of GOODS and COSMOS LAEs were drawn from different areas in the sky, the effect of biased cosmic variance seems unlikely, as could be identified in our images. The models from Dijkstra et al. (2007) may imply that our LAEs have moderate star formation rates.

\section{CONCLUSIONS AND SUMMARY}

The origin and escape path of $\operatorname{Ly} \alpha$ photons in highredshift galaxies has been a challenge to understand due 
to the complexity of the scattering and extinction process by the interstellar medium as well as absorption by intergalactic gas and dust. An investigation of both the spectroscopic and morphological properties of a large sample of LAEs is necessary to understand the link between the morphology of the rest-frame UV emission and that of the spectral profile shape of the Ly $\alpha$ emission line. In this paper we study the relationship between the asymmetry exhibited by the Ly $\alpha$ line profile and the rest-frame UV $(\sim 2300 \AA)$ morphology of a spectroscopically selected sample of 304 Ly $\alpha$ emitting sources at $3<z<7$ in the GOODS and COSMOS fields. Within the sample, $66 \mathrm{~B}$-dropouts, $43 \mathrm{~V}$-dropouts, and $7 i$-dropouts have been identified. The median redshift of the sample is $\langle z\rangle=4.55$.

Our main results include the following:

- There is a large spread in skewness values for all sources at low axial ratio values, and this spread decreases as the elongation of the galaxies increases. This trend is consistent with the expectations that outflows are present. Models have shown that in the case of a galaxy with a central monochromatic source and an expanding shell of gas, Ly $\alpha$ photons would undergo backscatterings on their escape path and broaden the line profile, and this effect would be most pronounced in the face-on cases where the outflowing gas is directly along our line of sight. If outflows were present, and indeed recent literature seems to suggest that they are commonplace in these high-redshift star-forming systems, the expected orientation of the galactic winds as well as the broadening effect on the spectral line are both consistent with the observed trend between Ly $\alpha$ asymmetry and UV morphology in this work.

- In the absence of outflows, we explore the Ly $\alpha$ line profile shapes predicted by radiative transfer models that probe different rotation velocities and distributions of the ionizing sources at various optical depths and viewing angles. With the simplifying assumption that these LAEs are intrinsically round and symmetric disks, rotation alone is insufficient in explaining the observed trend between skewness and axial ratio. In order to account for the relative abundance of asymmetric line profiles in the low and high axial ratio cases, consideration of optical depths is also invoked. The optical depth probed may directly indicate the amount of intervening gas, and thus, absorption and scattering of Ly $\alpha$ photons into the line wings, along the line of sight. The enhancement of the double-peaked feature in the Ly $\alpha$ line would cause its profile shape to appear symmetric at high $a / b$ values.

- Both the Ly $\alpha$ line profile shape and the axial ratio of the rest-frame UV emission may potentially be redshift dependent due to IGM absorption and diminishing sensitivity, respectively. Results from our Spearman rank correlation tests show that neither axial ratio nor Ly $\alpha$ skewness has any statistical dependence on redshift, so any variations we see in each of the spectral and morphological properties are likely intrinsic to the galaxies. We also checked for any potential effect on source size,
Ly $\alpha$ flux, skewness, and axial ratio that the SNR of the Ly $\alpha$ line may have, and found no correlation.

We thank the referee for a thorough review and many helpful suggestions for improving the paper. VU acknowledges helpful discussions of the various statistical tests with CWK Chiang, as well as partial funding support from the Thirty Meter Telescope International Observatory and the UC Chancellor's Postdoctoral Fellowship Program. The work of DS was carried out at Jet Propulsion Laboratory, California Institute of Technology, under a contract with NASA. The data presented herein were obtained at the W.M. Keck Observatory, which is operated as a scientific partnership among the California Institute of Technology, the University of California and the National Aeronautics and Space Administration. The Observatory was made possible by the generous financial support of the W.M. Keck Foundation. The authors wish to recognize and acknowledge the very significant cultural role and reverence that the summit of Mauna Kea has always had within the indigenous Hawaiian community. We are most fortunate to have the opportunity to conduct observations from this mountain.

\section{REFERENCES}

Ahn, S.-H., Lee, H.-W., \& Lee, H. M. 2001, ApJ, 554, 604 -. 2003, MNRAS, 340, 863

Beckwith, S. V. W., Stiavelli, M., Koekemoer, A. M., et al. 2006, AJ, 132,1729

Bertin, E., \& Arnouts, S. 1996, A\&AS, 117, 393

Bouwens, R. J., Illingworth, G. D., Oesch, P. A., et al. 2010, ApJ, 709, L133

Bunker, A. J., Moustakas, L. A., \& Davis, M. 2000, ApJ, 531, 95

Capak, P., Aussel, H., Ajiki, M., et al. 2007, ApJS, 172, 99

Christensen, L., Sánchez, S. F., Jahnke, K., et al. 2004, A\&A, 417,487

Dey, A., Spinrad, H., Stern, D., Graham, J. R., \& Chaffee, F. H. 1998, ApJ, 498, L93

Dickinson, M. 1998, in The Hubble Deep Field, ed. M. Livio, S. M. Fall, \& P. Madau, 219

Dijkstra, M., Lidz, A., \& Wyithe, J. S. B. 2007, MNRAS, 377, 1175

Ellis, R., Santos, M. R., Kneib, J.-P., \& Kuijken, K. 2001, ApJ, 560, L119

Faber, S. M., Phillips, A. C., Kibrick, R. I., et al. 2003, in Society of Photo-Optical Instrumentation Engineers (SPIE) Conference Series, Vol. 4841, Instrument Design and Performance for Optical/Infrared Ground-based Telescopes, ed. M. Iye \& A. F. M. Moorwood, 1657-1669

Ferguson, H. C., Dickinson, M., Giavalisco, M., et al. 2004, ApJ, 600, L107

Fosbury, R. A. E., Villar-Martín, M., Humphrey, A., et al. 2003. ApJ, 596, 797

Frye, B., Broadhurst, T., \& Benítez, N. 2002, ApJ, 568, 558

Garavito-Camargo, J. N., Forero-Romero, J. E., \& Dijkstra, M. 2014, ArXiv e-prints: 1408.2561, arXiv:1408.2561

Giavalisco, M., Ferguson, H. C., Koekemoer, A. M., et al. 2004, ApJ, 600, L93

Grogin, N. A., Kocevski, D. D., Faber, S. M., et al. 2011, ApJS, 197, 35

Guo, Y., Ferguson, H. C., Giavalisco, M., et al. 2013, ApJS, 207, 24

Hu, E. M., Cowie, L. L., Barger, A. J., et al. 2010, ApJ, 725, 394

Kashikawa, N., Shimasaku, K., Malkan, M. A., et al. 2006, ApJ, 648, 7

Koekemoer, A. M., Faber, S. M., Ferguson, H. C., et al. 2011, ApJS, 197, 36 
Kornei, K. A., Shapley, A. E., Martin, C. L., et al. 2012, ApJ, 758,135

Kulas, K. R., Shapley, A. E., Kollmeier, J. A., et al. 2012, ApJ, 745,33

Laursen, P., Sommer-Larsen, J., \& Razoumov, A. O. 2011, ApJ, 728,52

Law, D. R., Steidel, C. C., Shapley, A. E., et al. 2012, ApJ, 759, 29

Leauthaud, A., Massey, R., Kneib, J.-P., et al. 2007, ApJS, 172, 219

Mallery, R. P., Mobasher, B., Capak, P., et al. 2012, ApJ, 760, 128

Mosleh, M., Williams, R. J., Franx, M., et al. 2012, ApJ, 756, L12

Murayama, T., Taniguchi, Y., Scoville, N. Z., et al. 2007, ApJS, 172,523

Ouchi, M., Shimasaku, K., Okamura, S., et al. 2004, ApJ, 611, 685

Ouchi, M., Shimasaku, K., Furusawa, H., et al. 2010, ApJ, 723, 869

Pénin, A., Cuby, J.-G., Clément, B., et al. 2015, A\&A, 577, A74

Pettini, M., Shapley, A. E., Steidel, C. C., et al. 2001, ApJ, 554, 981

Rauch, M., Miralda-Escudé, J., Sargent, W. L. W., et al. 1997, ApJ, 489, 7

Ravindranath, S., Giavalisco, M., Ferguson, H. C., et al. 2006, ApJ, 652, 963
Schenker, M. A., Ellis, R. S., Konidaris, N. P., \& Stark, D. P. 2013, ApJ, 777, 67

Scoville, N., Aussel, H., Brusa, M., et al. 2007, ApJS, 172, 1

Shapley, A. E., Steidel, C. C., Pettini, M., \& Adelberger, K. L. 2003, ApJ, 588, 65

Shioya, Y., Taniguchi, Y., Sasaki, S. S., et al. 2009, ApJ, 696, 546

Stark, D. P., Ellis, R. S., Bunker, A., et al. 2009, ApJ, 697, 1493

Stark, D. P., Ellis, R. S., \& Ouchi, M. 2011, ApJ, 728, L2

Stark, D. P., Schenker, M. A., Ellis, R., et al. 2013, ApJ, 763, 129

Steidel, C. C., Erb, D. K., Shapley, A. E., et al. 2010, ApJ, 717, 289

Steidel, C. C., Giavalisco, M., Pettini, M., Dickinson, M., \& Adelberger, K. L. 1996, ApJ, 462, L17

Stern, D., Dey, A., Spinrad, H., et al. 1999, AJ, 117, 1122

Tapken, C., Appenzeller, I., Noll, S., et al. 2007, A\&A, 467, 63

Vanzella, E., Giavalisco, M., Dickinson, M., et al. 2009, ApJ, 695, 1163

Venemans, B. P., Röttgering, H. J. A., Miley, G. K., et al. 2005, A\&A, 431, 793

Verhamme, A., Schaerer, D., Atek, H., \& Tapken, C. 2008, A\&A, 491,89

Verhamme, A., Schaerer, D., \& Maselli, A. 2006, A\&A, 460, 397

Yamada, T., Matsuda, Y., Kousai, K., et al. 2012, ApJ, 751, 29 
Table 1

Spectroscopic Properties of LAEs

\begin{tabular}{lccccccc}
\hline \hline Source & RA (J2000) & Dec (J2000) & Type & Redshift & Redshift Flag & Skewness & Axial Ratio \\
\hline 1 & 53.14262 & -27.82654 & Bdrop & 3.569 & $\mathrm{~A}$ & $2.82 \pm 0.10$ & 1.38 \\
2 & 53.23541 & -27.86569 & Other & 4.705 & $\mathrm{~B}$ & $1.61 \pm 0.03$ & 1.35 \\
3 & 53.17774 & -27.82750 & Vdrop & 4.839 & $\mathrm{~A}$ & $1.45 \pm 0.04$ & 1.10 \\
4 & 53.07359 & -27.89222 & Bdrop & 3.701 & $\mathrm{~A}$ & $2.69 \pm 0.32$ & 1.14 \\
5 & 53.09012 & -27.95106 & Bdrop & 4.213 & $\mathrm{~A}$ & $2.01 \pm 0.04$ & 1.30 \\
6 & 53.08168 & -27.81108 & Bdrop & 3.715 & $\mathrm{~A}$ & $1.43 \pm 0.06$ & 1.15 \\
7 & 53.09518 & -27.74385 & Bdrop & 4.694 & $\mathrm{~A}$ & $1.20 \pm 0.12$ & 1.09 \\
8 & 53.06141 & -27.79965 & Other & 5.934 & $\mathrm{~A}$ & $6.15 \pm 1.53$ & 1.20 \\
9 & 53.06190 & -27.78507 & Other & 4.531 & $\mathrm{~A}$ & $4.35 \pm 0.35$ & 1.12 \\
10 & 53.08402 & -27.82344 & Bdrop & 3.655 & $\mathrm{~A}$ & $2.05 \pm 0.20$ & 2.27 \\
\hline
\end{tabular}

1 Table 1 is published in its entirety in the electronic edition of ApJ. A portion is shown here for guidance regarding its format and content.

${ }_{2}^{2}$ For the GOODS fields, quality flag 'A' indicates the most reliable redshifts, whereas the ' $\mathrm{B}$ ' redshifts are most likely correct. See text for details. Redshifts for the COSMOS sources have previously been published in Mallery et al. (2012).

Table 2

Summary of $B V i$ Dropouts

\begin{tabular}{lcccccc}
\hline \hline Type & \# in GOODS & \# in COSMOS & \# Total & $\left\langle z_{\text {spec }}\right\rangle$ & $\langle$ Skew $\rangle$ & $\langle a / b\rangle$ \\
\hline All & 130 & 174 & 304 & 4.55 & 1.55 & 1.26 \\
$B$-dropout & 57 & 9 & 66 & 3.93 & 1.58 & 1.21 \\
$V$-dropout & 20 & 23 & 43 & 4.82 & 1.68 & 1.22 \\
$i$-dropout & 6 & 1 & 7 & 5.61 & 1.81 & 1.25 \\
\hline
\end{tabular}


Table A1

Skewness Measurements of Simulated Spectra

\begin{tabular}{ccccc}
\hline \hline Noise Level & $s=0.5$ & $s=2.5$ & $s=4.5$ & $s=6.5$ \\
\hline Low: $10 \%$ of Amp. & $\langle s\rangle=0.51$ & $\langle s\rangle=1.72$ & $\langle s\rangle=2.60$ & $\langle s\rangle=4.56$ \\
High: $20 \%$ of Amp. & $\bar{s}=0.40 \pm 0.33$ & $\bar{s}=1.75 \pm 0.18$ & $\bar{s}=2.87 \pm 0.90$ & $\bar{s}=4.85 \pm 1.07$ \\
& $\bar{s}=0.41 \pm 0.13$ & $\bar{s}=1.66 \pm 0.32$ & $\bar{s}=2.65 \pm 1.61$ & $\bar{s}=4.81 \pm 2.74$ \\
\hline
\end{tabular}

\section{APPENDIX}

\section{A. TESting the Robustness of OUR Line-FitTing PROGRAM}

To test the robustness of our skewness measurements from our line-fitting procedure, particularly at the low S/N level, we show here an in-depth simulation exercise. Using the Gaussian equation given in Eqn. 4 we simulated a suite of Gaussian lines with varying skewness $(s=0.5,2.5,4.5$, and 6.5$)$ at two different noise levels (see examples in Fig. A1). Random noise has been added to the simulated spectra with fixed amplitude, centroid, line width, and continuum level. This is done so that the effect of noise on skewness measurement can be isolated. For our final set of simulations, 10 spectra have been generated at each of four skewness and two noise levels, totaling a suite of 80 simulated spectra to be tested with our line-fitting program.
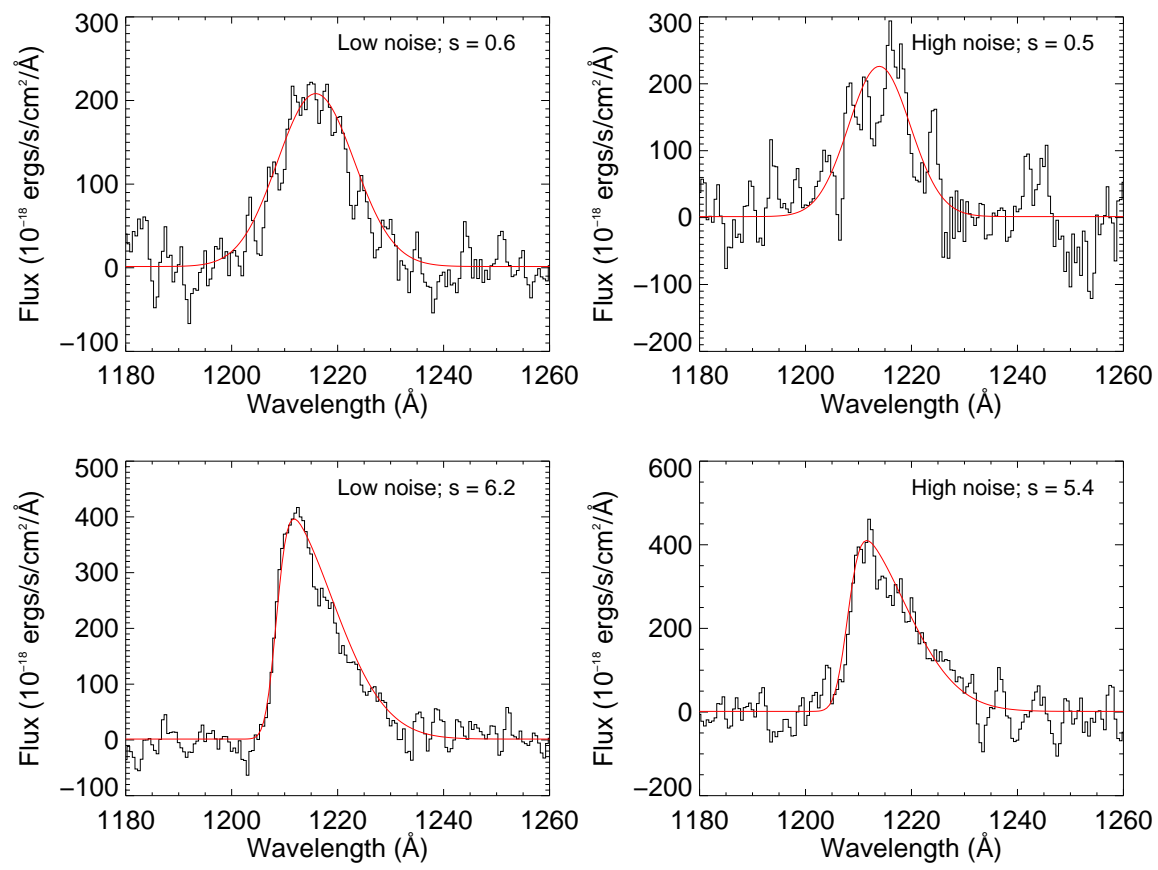

Figure A1. Examples of simulated spectra (black) with low and high intrinsic skewness and noise levels, respectively. The panels in the top row have intrinsic skewness of $s=0.5$, and those in the bottom row have $s=6.5$. The spectra have been smoothed by a width of 3 pixels for presentation purposes. The skewed Gaussian fits are shown in red.

Our main fitting results from the simulation exercise is summarized in Table A1 and Fig. A2 In general, the measured skewness values fall short of the corresponding intrinsic parameter across the span of the skewnesses probed. For low skewness values, noise level plays a less significant role in affecting the resulting fits. Lines that are intrinsically more skewed tend to have their skewness underestimated as noise increases, though the differences in the median and mean skewnesses between low and high noise levels are marginal (up to $8 \%$ at $s=6.5$ ).

These results imply that our skewness values as measured from our line-fitting program may be underestimated, depending on the noise level. Given the lack of trends seen in skewness versus Ly $\alpha$ line S/N for our sample (Fig. 7), we believe that any systematics that may be inherent in our fitting procedure would not affect our overall conclusions and in particular, Fig. 5, in the paper. In fact, this exercise offers confidence that our highly asymmetric data points are indeed real, preserving the discussions of the major results in this work. 


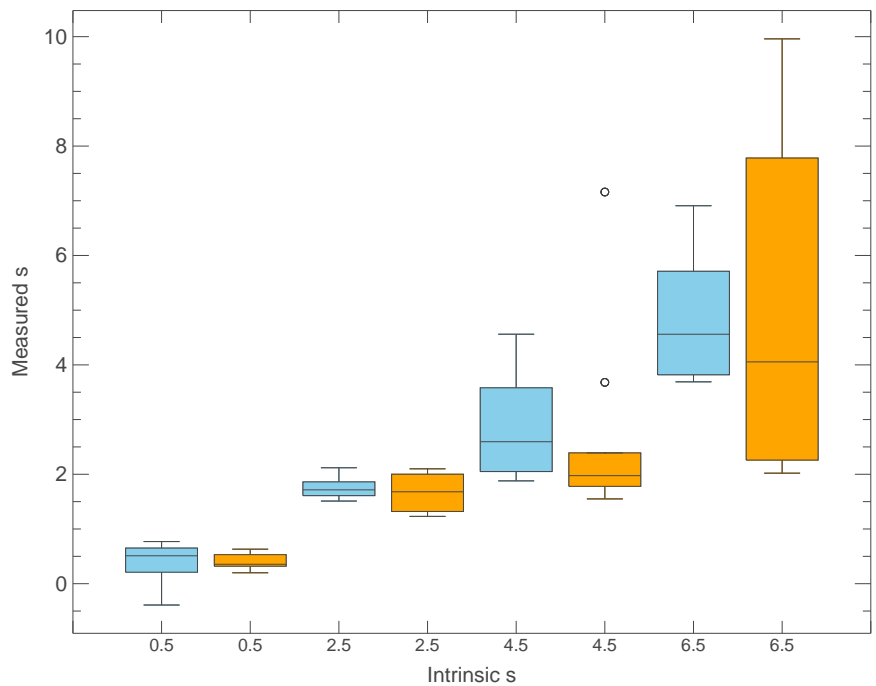

Figure A2. A box and whisker plot showing the interquartile $\left(25^{t h}\right.$ and $\left.75^{t h}\right)$ and $\max / \min$ ranges of the measured skewness of the simulated spectra in low (pale blue) and high (orange) noise bins. Over all, it appears that the measured skewness falls short of the corresponding intrinsic skewness. At low skewness values, noise level plays a less significant role in affecting the resulting fits. Lines that are more skewed tend to be fitted with a lower skewness value as noise increases. A couple outlier points are identified with small circles. 